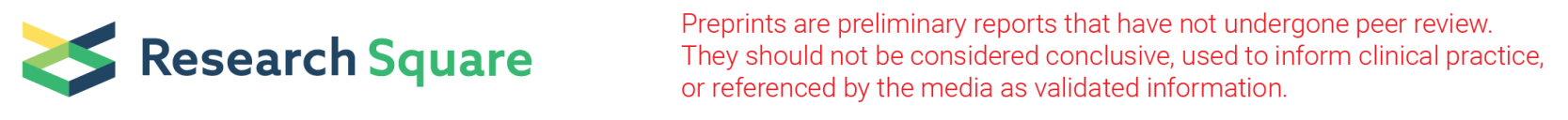

\title{
The impact of pulse oximetry and Integrated Management of Childhood Illness (IMCI) training on antibiotic prescribing practices in rural Malawi: a mixed-methods study
}

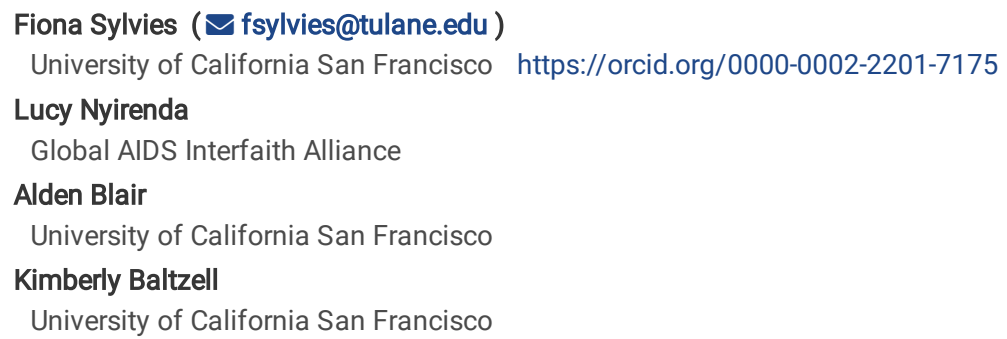

Research

Keywords: non-malarial fever, pulse oximeter, Integrated Management of Childhood Illnesses (IMCI), pediatric pneumonia, antibiotic resistance, mobile health clinic

Posted Date: April 22nd, 2020

DOI: https://doi.org/10.21203/rs.3.rs-23789/v1

License: (c) (i) This work is licensed under a Creative Commons Attribution 4.0 International License. Read Full License

Version of Record: A version of this preprint was published at PLOS ONE on November 19th, 2020. See the published version at https://doi.org/10.1371/journal.pone.0242440. 


\section{Abstract}

Background. The misdiagnosis of non-malarial fever in sub-Saharan Africa has contributed to the significant burden of pediatric pneumonia and the inappropriate use of antibiotics in this region. This study aims to assess the impact of 1) portable pulse oximeters and 2) Integrated Management of Childhood IIIness $(\mathrm{IMCl})$ continued education training on the diagnosis and treatment of non-malarial fever amongst pediatric patients being treated by the Global AIDS Interfaith Alliance (GAIA) in rural Malawi.

Methods. This study involved a logbook review to compare treatment patterns between five GAIA mobile clinics in Mulanje, Malawi during April-June 2019. An intervention study design was employed with four study groups: 1) 2016 control, 2) 2019 control, 3) IMCl-only, and 4) IMCl and pulse oximeter. A total of 3,504 patient logbook records were included based on these inclusion criteria: age under five years, febrile, malaria-negative, and treated during the dry season. A qualitative questionnaire was distributed to the participating GAIA providers. Fisher's Exact Testing and odds ratios were calculated to compare the prescriptive practices between each study group and reported with $95 \%$ confidence intervals.

Results. The pre- and post-exam scores for the providers who participated in the IMCI training showed an increase in content knowledge and understanding $(\mathrm{p}<0.01)$. The antibiotic prescription rates in each study group were $75 \%$ (2016 control), $85 \%$ (2019 control), $84 \%$ (IMCl only), and $42 \%$ (IMCl + pulse oximeter) $(p<0.01)$. An increase in pneumonia diagnoses was detected for patients who received pulse oximeter evaluation with an oxygen saturation $<95 \%$ ( $<<0.01)$. No significant changes in antibiotic prescribing practices were detected in the IMCl-only group $(p>0.05)$. However, provider responses to the qualitative questionnaires indicated alternative benefits of the training including improved illness classification and increased provider confidence.

Conclusion. Clinics that implemented both the IMCl course and pulse oximeters exhibited a significant decrease in antibiotic prescription rates, thus highlighting the potential of this tool in combatting antibiotic overconsumption in low-resource settings. Additionally, the pulse oximeters demonstrated the capacity to improve detection of pediatric pneumonia. GAIA staff appreciated the IMCl continued education training, however it did not appear to significantly impact antibiotic prescription rates and/or pneumonia diagnosis.

\section{Background And Significance}

While the diagnosis and treatment of malaria has greatly improved due to the advent of rapid diagnostic tests, non-malarial causes of fever still represent a significant burden of disease throughout sub-Saharan Africa (SSA). ${ }^{1}$ Within this region, non-malarial fever (NMF) is critical to address in Malawi, particularly amongst patients under the age of five. ${ }^{2}$ Of the many causes of NMF in Malawi, pneumonia accounts for $13 \%$ of deaths amongst children under the age of five, and is thus essential to accurately diagnose and treat. ${ }^{3}$ It is estimated that one in five pediatric deaths due to pneumonia could be avoided if providers showed stronger adherence to existing diagnostic guidelines and interventions. ${ }^{4}$

Not only does the inaccurate diagnosis of pneumonia lead to adverse patient outcomes, but it also poses a major public health concern due to the subsequent overuse of antibiotic prescriptions. Due to the vague presentation of febrile illness in pediatric patients, Malawian providers tend to over-rely on the

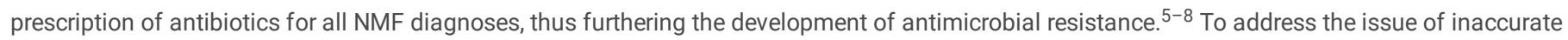
diagnoses, additional testing is needed to help providers differentiate febrile patients with or without pneumonia, so that they can determine when antibiotics can be safely distributed or withheld. In the context of low-resource settings, this diagnostic methodology should ideally be cost-effective, function independently of electricity, require minimal training, and provide results that are easy to interpret. ${ }^{9}$

Two such diagnostic resources include 1) portable pulse oximeters and 2) the Integrated Management of Childhood IIIness (IMCI) continued education courses. Despite being a standard tool in most developed healthcare systems, the pulse oximeter is not widely available in Malawian health facilities, a constraint that is especially exacerbated in rural areas. ${ }^{10,11}$ While studies regarding the use of pulse oximeters and/or IMCl continued education courses are expanding in hospital settings, little is known about the impact of implementing either intervention on antibiotic prescriptive rates in alternative sources of health care delivery, such as mobile clinics. Characteristics inherent in mobile clinics, namely their lack of geographical permanence, may increase providers' over-reliance on antibiotics due to concerns of patients' febrile symptoms worsening during the time it takes for the clinic to return to a given community. ${ }^{5}$ As $85 \%$ of the Malawian population resides in rural areas, improving the capacity of mobile clinics to diagnose and treat NMF amongst these hard-to-reach communities is crucial. ${ }^{12}$

Addressing these issues are in the forefront of the concerns for providers working for the Global AIDS Interfaith Alliance (GAIA) in Mulanje, Malawi. GAIA is a nonprofit organization which aims to increase healthcare accessibility amongst rural communities based in southern Malawi through the use of mobile clinics that rotate between the many villages throughout this region. ${ }^{13}$ These GAIA providers operate under tremendously challenging conditions, given that each mobile clinic team typically includes one clinical officer, two nurses and one nurse aid, seeing an average of 160 patients per day. ${ }^{13}$ This hardship is further compounded by underdeveloped roads, seasonal tropical storms and frequent floods that burden southern Malawi. ${ }^{14}$ Given the transient nature of these clinics, GAIA represented an ideal platform to assess the impact of an implementation of both portable pulse oximeters and IMCl continued education courses. This study aims to understand the impact of these interventions, both individually and together, on pediatric fever diagnosis and prescribing practices in rural Malawi.

\section{Methods}

This study is a continuation of recent research regarding NMF diagnosis in Malawi which concluded that rapid point-of-care tests were needed to help providers accurately diagnose febrile patients in order to make informed decisions when prescribing antibiotics. ${ }^{6,15}$ Specifically, this quantitative study used a logbook review to compare provider use of the IMCI guidelines to the use of the IMCl guidelines in conjunction with pulse oximeters. 
An intervention study design was employed with four comparison groups; 1) logbook review of prescribing practices from April-July 2016 prior to any interventions, referred to as control group 1;2) logbook review of prescribing practices from April-July 2019 in clinics in which neither intervention was implemented, referred to as control group 2 to account for changes in national protocols from 2016-2019;3) logbook review from April-July 2019 of prescribing practices at clinics receiving IMCl continued education training only; and 4) logbook review of prescribing practices from April-July 2019 at clinics receiving both $\mathrm{IMCl}$ continued education training and pulse oximetry training (Table 1). The data collection period took place over six weeks (May 6, $2019-$ June 14, 2019).

Prior to the start of the study period, GAIA providers from five mobile clinics participated in one of two scheduled IMCl continued education courses held in March and April, 2019. The structure of this five-day course involved three days of theory-based learning in a classroom setting, followed by two days of practical-based learning in a pediatric clinical setting (Mulanje District Hospital). These courses were attended by 15 providers total, including both nurses and clinical officers. The participating providers were asked to take a pre-exam prior to the course, followed by a post-exam after completion of the training course. Each mobile clinic provider was assigned to a specific mobile clinic, thus the crossover of providers between mobile clinics did not occur. Clinic site 3 was originally intended to be included in the intervention group which employed both the IMCl continued education course and the pulse oximeters. However, due to scheduling conflicts, the clinical officer from this clinic was unable to attend the pulse oximeter training, thus excluding clinic site $3 \mathrm{from}$ the IMCl/pulse oximetry intervention group. Clinical officers and nurses from clinic sites 4 and 5 did participate in the training course, thus these two clinic sites comprised the $\mathrm{IMCl} /$ pulse oximetry intervention group.

Participating providers then attended a brief training course in May 2019 on the use of the pulse oximeters. Training included a demonstration in the use of pulse oximeters followed by discussion with the providers. Providers in the intervention group receiving pulse oximeters were instructed to use $95 \%$ oxygen saturation as a cut-off for antibiotic use. This threshold is based on previously established indicators of pneumonia: oxygen saturation < $95 \%$, lung crackles on auscultation, fever $>37.8^{\circ} \mathrm{C}$, and pulse rate $>100$ beats per minute. ${ }^{16}$ Use of the pulse oximeter was an adjunct to clinical observation and IMCl guidelines to arrive at a diagnostic and treatment decision. Both standard and neonatal pulse oximeters were distributed to group 4 (clinic sites 4 and 5 ) (Table 1).

A total of 3,504 patient logbook records were included for analysis. Review of the GAIA patient logbooks allowed for assessment of the implementation of and adherence to $\mathrm{IMCl}$ guidelines and pulse oximeter parameters, and the resulting diagnosis and treatment of pediatric patients with NMF. Quantitative data extracted from logbooks included: clinic site, patient age, date, diagnosis, and drugs prescribed. For the two clinics that implemented pulse oximeters, data were also recorded on whether a pulse oximeter was used and the resulting oxygen saturation measurement. To evaluate changes in the use of the pulse oximeter in the IMCl/pulse oximetry group over the six-week study period, data were collected regarding the percentage of U-5 NMF patients that were evaluated with a pulse oximeter during this time. All quantitative data were collected and stored using Redcap (version 9.1.0), a secure online platform designed for managing surveys and databases. ${ }^{17}$ The medical records were stored in hand-written logbooks that were securely stored in the GAIA clinical offices in Mulanje and Limbe, Malawi.

After the data collection period, brief qualitative questionnaires were distributed to the GAIA clinical staff regarding their opinion of the IMCI training course and/or pulse oximeters. The questionnaire was given verbally in English and recorded, with provider consent. The names of the providers were not recorded during the questionnaire, but their position as either clinical officer, nurse, or nurse aide was documented.

\section{Data Analysis}

To assess for changes in antibiotic prescriptions, the proportion of U-5 patients presenting with NMF who were prescribed antibiotics was compared among the four study groups. These proportions reflected provider adherence to either intervention when deciding to distribute or withhold antibiotics for NMF patients. To assess for differences in the prescriptive practices among the four study groups, Fisher's exact test and simple logistic regressions were used. Adherence to the IMCI guidelines was determined by assessing whether the diagnosis made by the provider was followed by the IMCI recommended treatment, as reflected in the GAIA patient logbooks. Adherence to the pulse oximetry protocol was evaluated based on the percentage of patients who received a normal pulse oximeter measurement of ${ }^{3} 95 \%$ who then received the protocol's recommended treatment of basic analgesics. P-values less than 0.05 were considered significant. Odds ratios were also reported with corresponding $95 \%$ confidence intervals to provide both the strength and direction of association. All analyses were performed using the standard statistical analysis package R (version 3.5.1). ${ }^{18}$

The provider responses to the open-ended questionnaires were designed to help the GAIA management staff understand the experiences of the providers throughout the study and thus were considered supplemental to this study. Therefore, strict qualitative analysis methods were not employed for this portion of the study.

\section{Results}

\section{Clinician population included in study}

As previously stated, the GAIA providers who participated in the IMCl continued education course were required to complete a knowledge assessment exam before and after the course. The averages of the pre-exam and post-exam scores for the participating providers are shown in Figure 1, categorized by clinical position. Both the IMCl-only and IMCl/pulse oximetry groups had nurses and clinical officers in attendance for one of the IMCl training courses, while clinics in the 2019 control group had only nurses attend the training. A significant increase in content knowledge and understanding was detected between the pre- and post-exam scores from IMCI training ( $p$-value $<0.01$ ). In comparing the scores of the nurses to the clinical officers, no significant difference was found in the average pre-scores, post-scores, or change in scores $(p>0.05)$. Nurse and clinical officer participation in the pulse oximeter training is outlined in Table 2. 


\section{Patient Demographics}

The demographics for the patient populations included in each of the four study groups can be found in Table 3. The 2016 control data consisted of 1,960 U-5 NMF patients seen by all five clinics. The sample sizes of the three remaining study groups were significantly smaller due to division of the five clinics according to which intervention was employed. The study group with the smallest sample size was that of the IMCl-only group, which only contained data from clinic site 3. A significant difference in the patient age distribution was detected among the four study groups $(p<0.05)$.

\section{Differences in antibiotic prescriptive patterns}

Overall, the odds of a patient receiving antibiotics in an intervention clinic that employed both IMCl training and pulse oximeters were 7.9 times less likely compared to a patient in the 2019 control group (95\% Cl 6.1-10.5), 7.3 times less likely than a patient in the IMCl-only group (95\% Cl 4.8-11.4), and 4.0 times less likely than a patient in the 2016 control group ( $95 \% \mathrm{Cl} 3.3-4.7)$. The significant difference in the antibiotic prescribing rates for U-5 NMF patients across the study groups can be seen in Figure 2, with rates of $75 \%$ in $2016,85 \%$ in the 2019 control group, $84 \%$ in the 2019 IMCl-only group, and $42 \%$ in the 2019 IMCI with pulse oximetry group $(\mathrm{p}<0.01)$.

\section{Pulse oximeter utilization}

Changes in the providers' use of the pulse oximeters for diagnosing U-5 NMF patients throughout the study period are shown in Figure 3. Utilization of the pulse oximeter was consistently higher in clinic site 5 during each week throughout the study period. However, clinic site 4 exhibited a steady increase in the use of the pulse oximeter over time.

A total of 795 patients were seen during the study period in intervention clinics using the pulse oximeter. Of these, 30\% ( $n=239$ ) received evaluation by pulse oximetry. Differences in patient diagnoses based on oxygen saturation cutoff can be seen in Figure 4. For patients who received pulse oximeter evaluation with a resulting oxygen saturation level greater than or equal to $95 \%$, the most common diagnosis was common cold (31\%), followed by acute respiratory infection (22\%), gastroenteritis (19\%), upper respiratory tract infection (18\%), and sepsis (9\%). Only $2 \%$ of patients with a normal oxygen saturation ( $\left.{ }^{3} 95 \%\right)$ were diagnosed with pneumonia. For patients who received pulse oximeter evaluation with a resulting oxygen saturation less than $95 \%$, the most common diagnosis was pneumonia (77\%), followed by acute respiratory infection (10\%), sepsis (5\%), bronchitis (4\%), and lower respiratory tract infection (4\%). A significant increase in pneumonia diagnoses was seen for pediatric patients with an oxygen saturation level less than $95 \%$ compared to patients who had an oxygen saturation of $95 \%$ or higher $(\mathrm{p}<0.01)$. For the remaining patients that did not receive pulse oximeter evaluation in the IMCl/pulse oximetry group, the most common diagnoses were acute respiratory infection (38\%), sepsis (19\%), gastroenteritis (17\%), and common cold (14\%). Of these patients, pneumonia represented $0.4 \%$ of all diagnoses.

\section{Qualitative provider interviews}

From the brief qualitative interviews conducted with the GAIA providers, key themes were identified regarding the perceived benefits and challenges of implementing either intervention (Figure 5). For quotes regarding provider opinion of either intervention, see Tables 4-5.

\section{Perceived benefits of IMCI continued education course}

Provider responses to the questionnaires exploring the IMCl continued education course were predominantly positive. The stepwise function of the IMCI guidelines was regarded as especially important in helping diagnose pediatric patients. Providers felt that the enhanced classification aspect of IMCI helped them to deconstruct the symptoms presented by each patient in order to generate a holistic diagnosis.

The providers also felt they had grasped the urgency of antibiotic resistance in Malawi and the need to be more conservative with the prescription of antibiotics for febrile pediatric patients. Several providers noted the increase in antibiotic resistance that they had witnessed firsthand through working with various patient populations over the years. This experience was described as stressful, as the providers relayed feeling "frustrated" and "defeated" when children would return to clinic without any improvement from previously administered antibiotics. Lastly, many reported an augmented sense of selfconfidence as a result of their participation in the IMCI training. By completing the IMCI training, many felt that they no longer had to speculate on a diagnosis.

\section{Perceived challenges of IMCI continued education course}

While participants did describe some challenges related to $\mathrm{IMCl}$ course participation, these challenges primarily involved issues regarding the scheduling of the course, rather than the content of the course itself. For future $\mathrm{IMCl}$ course arrangements, several providers felt that it would be beneficial to increase the duration of the course from five days to ten, with a larger portion of the training course devoted to the field-based practical component. One other criticism of the IMCl courses was that they had been scheduled in March and April, both overlapping with malaria season in Malawi. As this is the busiest season for the GAIA providers, several staff members recommended that future courses be arranged specifically in the dry season, so as to avoid major conflicts with the clinic schedules. 


\section{Perceived benefits of pulse oximeter utilization}

Similar to the responses regarding $\mathrm{IMCl}$ training, the providers perceived the impact of implementing pulse oximeters in the mobile clinics to be predominantly beneficial. The improved detection of pneumonia as indicated by low oxygen saturation on the pulse oximeter was deemed especially useful. The clinical officers from the $\mathrm{IMCl} /$ pulse oximetry group both believed that without the use of the pulse oximeter, many pneumonia patients may have been missed due to incomplete diagnostic evaluation. Due to the lack of diagnostic capacity in the mobile clinic setting and the rapid pace of patient flow in the mobile clinics, the providers noted the value of implementing a point-of-care device that can provide results in under 30 seconds.

In addition to increasing diagnostic capacity for identifying pneumonia, the clinical officers also felt that the pulse oximeters allowed them to be more conservative when prescribing antibiotics for febrile pediatric patients. This is supported by the quantitative finding which stated an approximately $50 \%$ decrease in antibiotic prescriptive rates seen in the clinics which implemented both pulse oximeters and IMCl continued education courses. The providers considered the $95 \%$ saturation threshold as a diagnostic cutoff to which they could easily adhere, thus simplifying their decision to prescribe antibiotics versus analgesics. The use of the pulse oximeters was also believed to increase the confidence of the patients' caretakers. Many of the parents and/or guardians of the pediatric patients were reported to have felt more assured when being told that antibiotics were not needed after having observed a normal oxygen saturation reading. Witnessing the use of this diagnostic technology left many caretakers with a feeling of relief in knowing that their child had been evaluated thoroughly in comparison to past clinical experiences.

\section{Perceived challenges of pulse oximeter implementation}

Several challenges were addressed regarding the use of the pulse oximeters in clinic. The primary challenge identified by the providers was that of applying the pulse oximeters to infants. Often times the very young children, particularly neonates and infants, demonstrated resistance to wearing the pulse oximeter. This resistance appeared to result from pediatric patients associating the use of the pulse oximeter with the use of mRDTs, and thus anticipating a painful experience. This resistance led providers to feel as though continuing to apply the pulse oximeter to the distressed child would be unethical. Additionally, one provider mentioned that on days in which the clinical officer was not working, it was difficult to incorporate the pulse oximeter into the diagnostic routine as the providers were already struggling to manage an under-staffed clinic. Lastly, several providers noted that issues related to the implementation of any new clinical protocol were observed, namely the missed documentation of the use of the pulse oximeter in the first few weeks of the study. However, these providers believed that the process of documenting pulse oximeter evaluations had improved over the six weeks.

\section{Discussion}

We investigated the impact of implementing two diagnostic interventions, pulse oximeters and IMCl continued education training, on the frequency of antibiotic prescriptions for U-5 NMF patients being treated in five mobile health clinics in Mulanje, Malawi. Data extracted from patient logbooks indicated a substantial reduction in antibiotic provision in clinics receiving both $\mathrm{IMCl}$ continued education training and portable pulse oximeters. However, there was no significant reduction in antibiotic prescribing practices among providers in the clinics which only implemented IMCI training. Data compiled from the provider interviews suggested additional benefits of provider participation in the $\mathrm{IMCl}$ training related to improved diagnostic confidence and personal empowerment.

To our knowledge, this study was one of the first to show a significant association between the use of pulse oximeters and decreased antibiotic prescriptions for febrile patients being treated in a rural mobile clinic setting. However, investigation into the impact of pulse oximeters on antibiotic prescribing practices has been expanding for urban outpatient settings in sub-Saharan Africa. ${ }^{19}$ The rate of antibiotics prescribed in the intervention group which utilized pulse oximeters was found to be approximately half of the rate exhibited in the three remaining study groups. This outcome is consistent with previous data which found that improved diagnosis of pneumonia amongst NMF patients through the implementation of diagnostic resources, such as point-of-care procalcitonin tests, can decrease unnecessary antibiotic consumption by $30-50 \% .{ }^{20,21}$ This finding indicates that the relatively simple and low-cost introduction of pulse oximeters into low-resource clinics that serve rural patient communities has the potential to greatly conserve antibiotic resources. Not only can this conservation help to deter the further development of resistance, but it can also benefit clinical organizations by allowing for increased financial savings and reallocation of funds given the higher cost of antibiotics compared to basic analgesics. ${ }^{22,23}$

Of the various benefits of utilizing the pulse oximeters in clinic, the subsequent improvement in pneumonia detection was universally commended by providers during the interviews. This qualitative finding, in conjunction with the significantly higher proportion of pneumonia diagnoses for patients with a low oxygen saturation measurement, represents a meaningful clinical improvement. Given the high burden that pneumonia represents amongst pediatric patients in Malawi, and elsewhere in SSA, and the ability of pulse oximeters to function in limited-resource settings, this device could greatly improve the diagnosis and treatment of pediatric pneumonia in rural areas. ${ }^{3}$

It is worth noting that the IMCl-only intervention group did not exhibit a significant decrease in the number and/or percentage of U-5 NMF patients prescribed antibiotics. This finding contradicts previous studies which found that IMCl courses increased providers' adherence to guidelines related to antibiotic distribution based on patient need. ${ }^{24}$ In the majority of the provider interviews, participants discussed the benefit of the IMCl course in training them to be more conservative with antibiotic prescriptions. However, such an outcome was not reflected in the analysis. One explanation for this inconsistency could be the lack of physical confirmation provided by the IMCl guidelines compared to the objective measurements offered by the pulse oximeters. It is understandable that when a pediatric patient presents with fever, despite no other physical signs of pneumonia as outlined in the IMCI guidelines, that a provider will still prescribe antibiotics to ensure the child's safety. Furthermore, many of the guardians who accompany U-5 children may expect to leave a clinic with antibiotics, it is also likely that they strongly insist on such a prescription. ${ }^{25,26}$ However, it was observed that for many guardians who were able to 
see that their child's oxygen saturation results fell within the healthy range, they felt confident enough in this "new" technology to return home with basic analgesics.

The lack of significant change in antibiotic prescriptions within the IMCl-only study group should not undermine the additional benefits that this course provided to the providers. Throughout the interviews, several providers conveyed a sense of empowerment after having completed the IMCl course. The significant increase in knowledge retention shown through their pre- and post-exam scores indicates the educational benefit of this course, both in theory and practice. This rise in diagnostic confidence is helpful in maintaining morale, and thus should not be overlooked. Furthermore, the augmented confidence that many providers experienced after completing this course represents a meaningful achievement given the lack of resources allotted in this setting.

As mentioned above, the most significant finding of this study is that antibiotics were significantly reduced in the intervention group that received both IMCI continued education training and use of the portable pulse oximeter. Both clinics in this intervention group had a clinical officer present during the study period. In Malawi, as with many countries around the world, nurses are often viewed as inferior to clinical officers and/or physicians, despite both completing three years of healthcare education. ${ }^{27,28}$ As such, when the clinical officer is present, he or she exclusively determines patient diagnoses and medication prescriptions. During the provider interviews, nurses from the IMCl-only clinic and the 2019 control clinics who had completed IMCl training expressed the need for decreasing unnecessary antibiotic prescriptions in order to deter further development of resistance. Yet, these clinics both exhibited high rates of antibiotic prescription. This finding indicates that regardless of training, it is ultimately the clinical officer who will govern significant changes in clinical output, a practice that limits the impact of positive trainings on other provider cadres. For an intervention to significantly impact diagnostic and prescriptive trends, nurses need to be given more responsibility and authority in the process of treating patients. Indeed, other studies in similar settings are beginning to show the significant impact of further empowering nurses in their clinical duties. ${ }^{29}$

\title{
Limitations
}

Due to the limited time allotted for the completion of this study, conducting patient follow-up to assess whether the diagnosis and treatment plan was appropriate was not possible. While the results from the pulse oximeter intervention group showed a significant benefit in terms of decreasing antibiotic prescriptions, it was not possible to conclude whether this decrease in antibiotics had any impact on patient wellbeing. However, several of the collaborators on this study recently published findings which showed that 14 days after U-5 febrile patients were evaluated in the same clinical setting, outcomes were stable to improved. ${ }^{6,30}$ Furthermore, given the significant increase in pneumonia diagnoses seen in the $\mathrm{IMCl} /$ pulse oximetry group, it is more likely that the wellbeing of pediatric patients was improved given the increased detection of pneumonia cases which may otherwise have been missed. The restriction of the IMCl-only intervention group to only one clinic site may have limited the evaluation of this intervention. Furthermore, it is possible that the significant impact of the pulse oximeter on prescriptive trends was a result of its implementation in conjunction with the IMCl training rather than as a stand-alone diagnostic intervention. Previous studies have found this combination of interventions to be optimal in improving the detection and treatment of pediatric pneumonia in resource-poor settings. ${ }^{31}$

\section{Conclusion}

In assessing the impact of multiple interventions on the diagnosis and treatment of pediatric non-malarial fever, this study found the use of simple pulse oximetry coupled with $\mathrm{IMCl}$ training helped significantly curb unnecessary antibiotic prescriptions in a low-resource setting. This finding points to the inclusion of pulse oximeters as a tool in the fight against antimicrobial resistance globally. Furthermore, the increase in pneumonia detection in clinics using pulse oximeters indicates the benefit of this device in identifying pneumonia among pediatric patients in similar settings. While the IMCl continued education course was not found to significantly influence antibiotic prescriptive trends, it was considered to be beneficial in stimulating provider confidence and empowerment. Additional investigation is needed to determine whether the success of the pulse oximeter can be replicated independently of IMCI training or if the combination of the two interventions provides the ideal balance of educational and physical resources to aid in diagnosing NMF pediatric patients. Future studies are also needed to closely follow patient outcomes when antibiotics are withheld and pre-determined oxygen saturation levels are used to guide provider treatment decisions.

\section{List Of Abbreviations}

\author{
NMF - non-malarial fever \\ IMCI - Integrated Management of Childhood IIInesses \\ GAIA - Global AIDS Interfaith Alliance \\ SSA - sub-Saharan Africa
}

\section{Declarations}

\section{Ethics Approval and Consent to Participate}

All participating GAIA providers signed informed consent. The patient information collected from the logbooks was anonymized, and no direct contact between the research team and the patients took place. As such, consent was waived for this study population. Ethical approval was obtained from both the 
University of California San Francisco (UCSF) Committee for Human Research (\#19-27452) and the Malawi National Health Sciences Research Committee (\# 19/03/2262) (NHSRC).

\section{Consent for Publication}

Not applicable.

Availability of data and materials

The datasets used and/or analyzed during the current study are available from the corresponding author on reasonable request.

\section{Competing Interests}

The authors declare that they have no competing interests.

Funding

This study was funded by the Global AIDS Interfaith Alliance and the Institute of Global Health Sciences of the University of California, San Francisco. The design of this study was led by a masters student at UCSF Institute for Global Health Sciences in collaboration with GAIA leadership.

\section{Author's Contributions}

FS was present in Malawi throughout the course of the study. Her roles included training the GAIA staff members on the use of the pulse oximeters, collecting data from the patient logbooks, distributing the provider questionnaires, conducting statistical analysis of the data, and writing the manuscript. LM oversaw all operations taking place within the clinics and served as a liaison between the UCSF research staff and the GAIA clinical providers. AB aided in the statistical analysis and edited the manuscript. $\mathrm{KB}$ organized the training courses for both the $\mathrm{IMCl}$ continued education as well as the pulse oximeter protocol and provided guidance throughout the study period regarding data collection and analysis.

\section{Acknowledgements}

The authors would like to thank the Global AIDS Interfaith Alliance (GAIA), with specific gratitude extended to the in-country team members who were particularly invested in this study; Joyce Jere, Nelson Khozomba, and Mphatso Phiri. Additional appreciation is also extended to Anna Muller, Elizabeth Geoffroy, and Ellen Schell for their support in editing the final manuscript.

\section{References}

1 D'Acremont V, Lengeler C, Genton B. Reduction in the proportion of fevers associated with Plasmodium falciparum parasitaemia in Africa: a systematic review. Malar J 2010; 9: 240.

2 Ministry of Health - National Malaria Control Program. Malawi Malaria Indicator Survey. 2017 https://dhsprogram.com/pubs/pdf/MIS28/MIS28.pdf (accessed Nov 19, 2018).

3 Black RE, Cousens S, Johnson HL, et al. Global, regional, and national causes of child mortality in 2008: a systematic analysis. Lancet 2010; 375: 1969-87.

4 Malawi Ministry of Health. Malawi Child Health Strategy for Survival and Health Development of Under-five Children in Malawi 2014-2020. Lilongwe, Malawi, 2013.

$5 \quad$ In personal communication with Dr. Kimberly Baltzell. 2018.

6 Baltzell KA, Bleakly Kortz T, Blair A, et al. A 14-day follow-up of adult non-malarial fever patients seen by mobile clinics in rural Malawi. 2018.

7 Collignon P, Athukorala PC, Senanayake S, Khan F. Antimicrobial resistance: the major contribution of poor governance and corruption to this growing problem. PLoS One 2015; 10: e0116746.

8 Oxford J, Kozlov R. Antibiotic resistance--a call to arms for primary healthcare providers. Int J Clin Pr Supp/ 2013; : 1-3.

9 WHO | Rapid diagnostic tests. WHO. 2018. https://www.who.int/malaria/areas/diagnosis/rapid_diagnostic_tests/en/ (accessed Dec 3, 2018).

10 McCollum ED, King C, Deula R, et al. Pulse oximetry for children with pneumonia treated as outpatients in rural Malawi. Bull World Health Organ 2016; 94: 893-902.

11 Ginsburg AS, Van Cleve WC, Thompson MIW, English M. Oxygen and pulse oximetry in childhood pneumonia: a survey of healthcare providers in resource-limited settings. J Trop Pediatr 2012; 58: 389-93.

12 The World Bank. Rural population of Malawi (\% of total population). 2017. https://data.worldbank.org/indicator/SP.RUR.TOTL.ZS?locations=MW (accessed Nov 19, 2018). 
16 Moore M, Stuart B, Little P, et al. Predictors of pneumonia in lower respiratory tract infections: 3C prospective cough complication cohort study. Eur Respir J 2017; 50: 1700434.

17 REDCap Research Electronic Data Capture. https://www.project-redcap.org/ (accessed July 15, 2019).

R: The R Project for Statistical Computing. https://www.r-project.org/ (accessed March 7, 2019).

19 Keitel K, Kagoro F, Samaka J, et al. A novel electronic algorithm using host biomarker point-of-care tests for the management of febrile illnesses in Tanzanian children (e-POCT): A randomized, controlled non-inferiority trial. PLoS Med 2017; 14: e1002411.

20 Burkhardt O, Ewig S, Haagen U, et al. Procalcitonin guidance and reduction of antibiotic use in acute respiratory tract infection. Eur Respir J 2010; 36 : $601-7$.

21 Pletz MW, Rohde GG, Welte T, Kolditz M, Ott S. Advances in the prevention, management, and treatment of community-acquired pneumonia. F1000Research 2016; 5. DOI:10.12688/f1000research.7657.1.

22 Desalegn AA. Assessment of drug use pattern using WHO prescribing indicators at Hawassa University teaching and referral hospital, south Ethiopia: a cross-sectional study. BMC Health Serv Res 2013; 13: 170.

23 Cameron A, Ewen M, Ross-Degnan D, Ball D, Laing R. Medicine prices, availability, and affordability in 36 developing and middle-income countries: a secondary analysis. 2008. DOI:10.1016/S0140.

24 Stellenberg E, Van Zyl M, Eygelaar J. Knowledge of community care workers about key family practices in a rural community in South Africa. African J Prim Heal care Fam Med 2015; 7: 892.

25 Björnsdóttir I, Hansen EH. Intentions, strategies and uncertainty inherent in antibiotic prescribing. Eur J Gen Pract 2002; 8: 18-24.

26 Little P, Dorward M, Warner G, Stephens K, Senior J, Moore M. Importance of patient pressure and perceived pressure and perceived medical need for investigations, referral, and prescribing in primary care: nested observational study. BMJ 2004; 328: 444.

27 Muula AS. Case for clinical officers and medical assistants in Malawi. Croat Med J 2009; 50: 77-8.

28 GOVERNMENT OF MALAWI MINISTRY OF HEALTH. Nurse/Midwife Training Operational Plan Field Assessments, Analysis and Scale-up Plans for Nurse Training Institutions. 2011 https://www.k4health.org/sites/default/files/Malawi_National Nurse Training Operational Plan.pdf (accessed July 8, 2019).

29 Manojlovich M. Power and Empowerment in Nursing: Looking Backward to Inform the Future. Online J Issues Nurs $2007 ; 12$.

http://ojin.nursingworld.org/mainmenucategories/anamarketplace/anaperiodicals/ojin/tableofcontents/volume122007/no1jan07/lookingbackwardtoinformt (accessed July 17, 2019).

30 Kortz TB, Blair A, Scarr E, et al. Characterizing Pediatric Non-Malarial Fever and Identifying the At-Risk Child in Rural Malawi. Glob Pediatr Heal 2018; 5: $2333794 \times 17750415$.

31 Floyd J, Wu L, Hay Burgess D, Izadnegahdar R, Mukanga D, Ghani AC. Evaluating the impact of pulse oximetry on childhood pneumonia mortality in resource-poor settings. Nature 2015; 528: 53-9.

\section{Tables}

Due to technical limitations the tables area available as a download in the Supplementary Files.

\section{Figures}


Pre-exam score (\%) $\quad$ Post-exam score (\%)

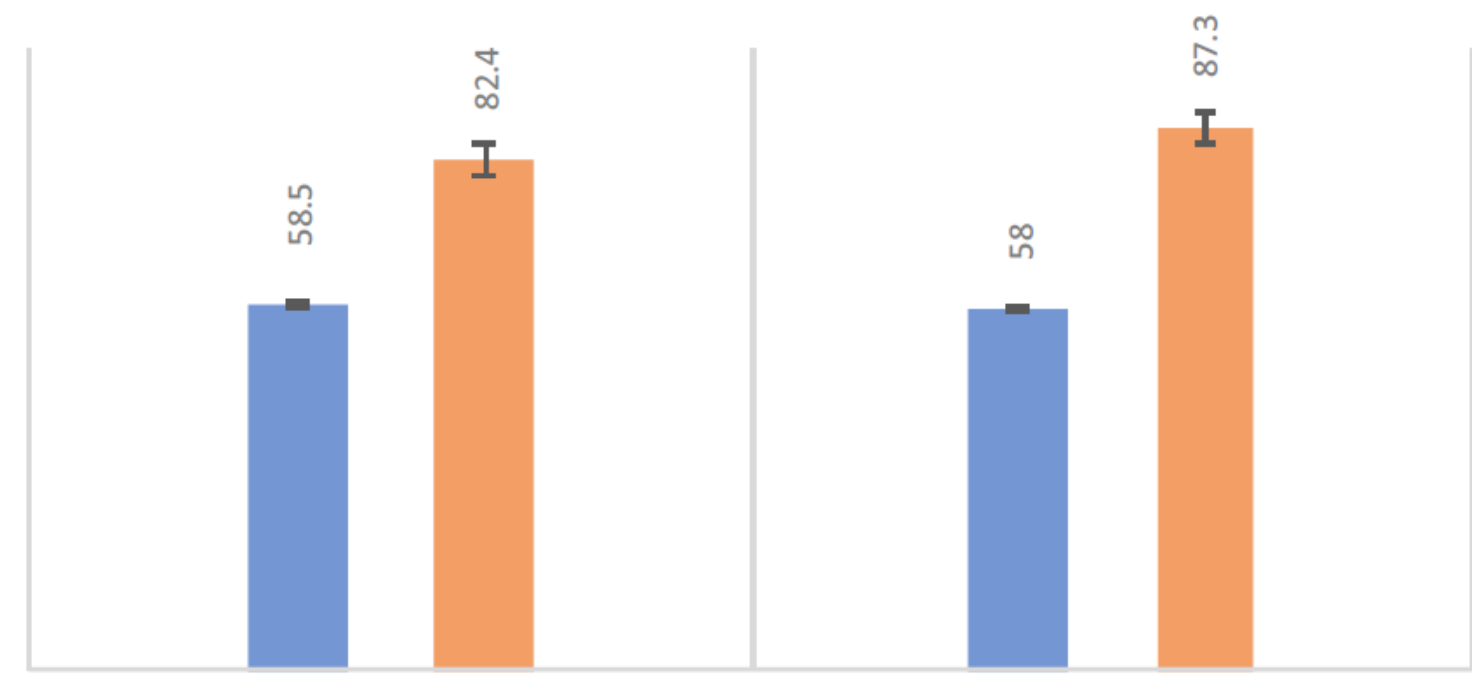

Nurse

Clinical Officer

Figure 1

Average pre- and post- exam scores for the 15 GAIA providers who participated in either the first or second IMCl continued education training course (March 48 or April 1-5 respectively) categorized by clinical position as either nurse or clinical officer.

90

80

70

60

50

40

30

20

10

0

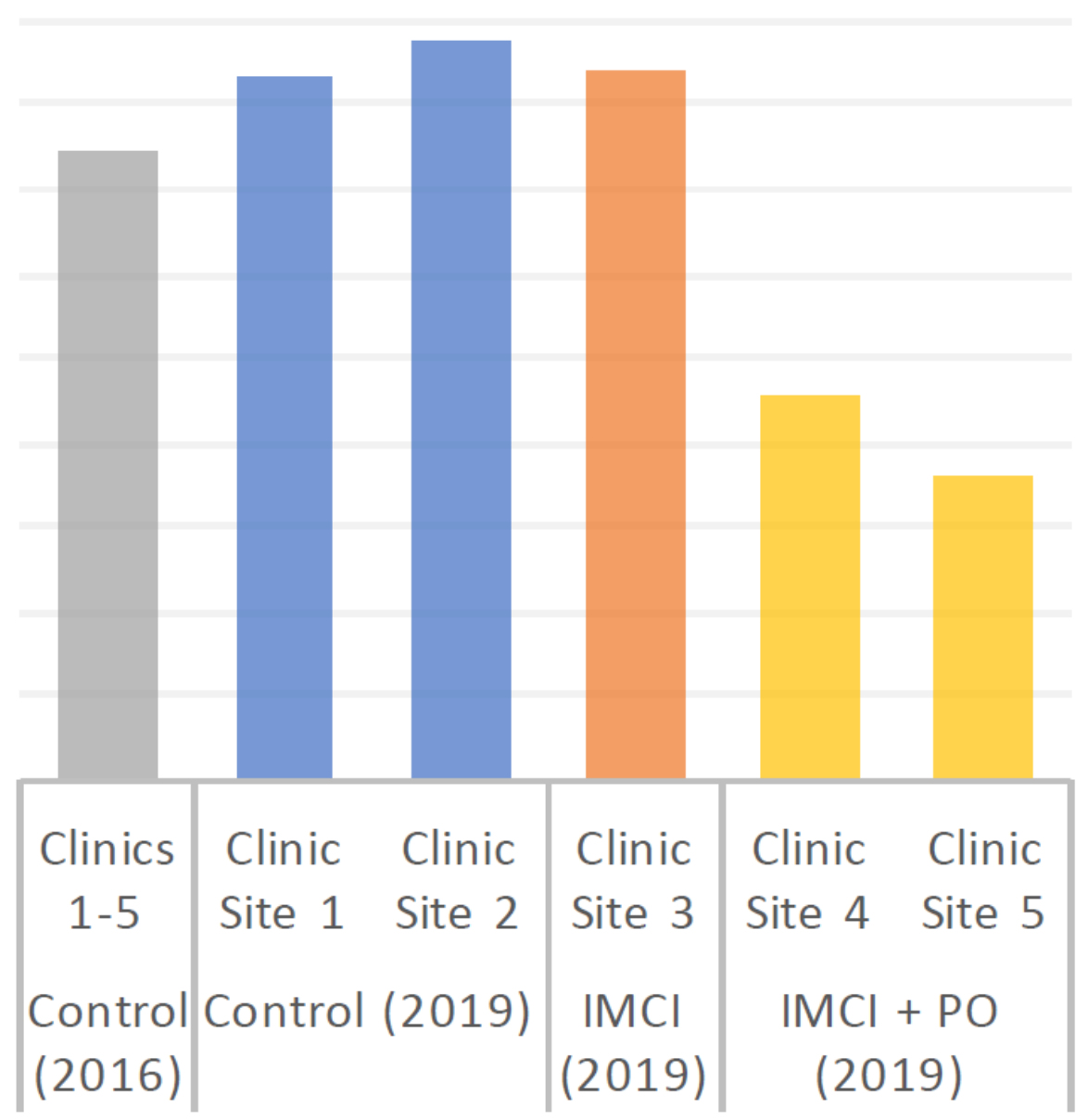

Figure 2 
Percentage of U-5 NMF patients prescribed antibiotics per clinic site within each study group: Control 2016, Control 2019, IMCl-only, and IMCl/pulse oximeter (PO).

\section{$70 \%$}
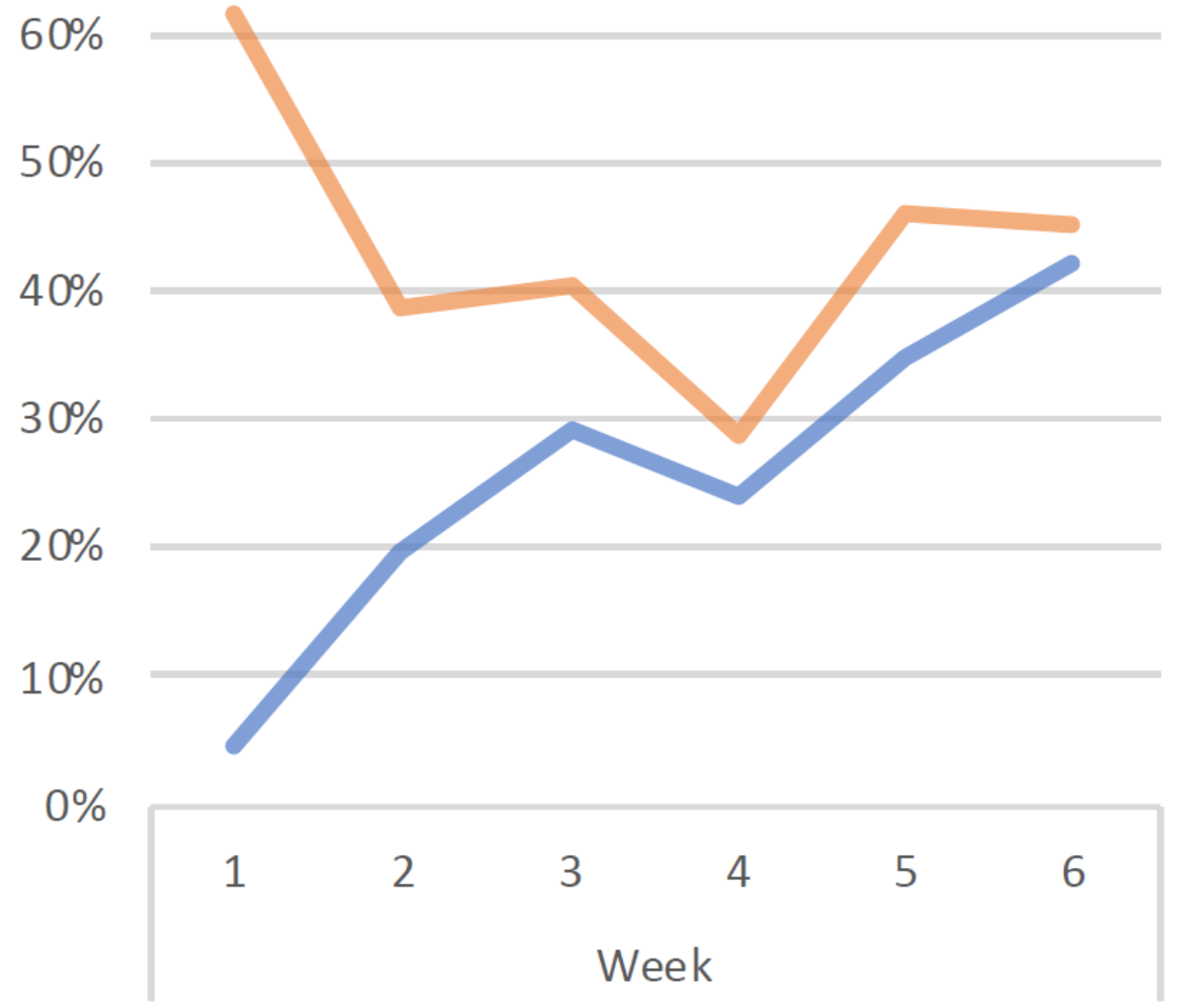

\section{Clinic Site $4 \longrightarrow$ Clinic Site 5}

\section{Figure 3}

Percentage of U-5 NMF patients evaluated with a pulse oximeter in clinic sites 4 and 5 over the six-week study period. 


\section{OXYGEN SATURATION $\geq 95 \%$}

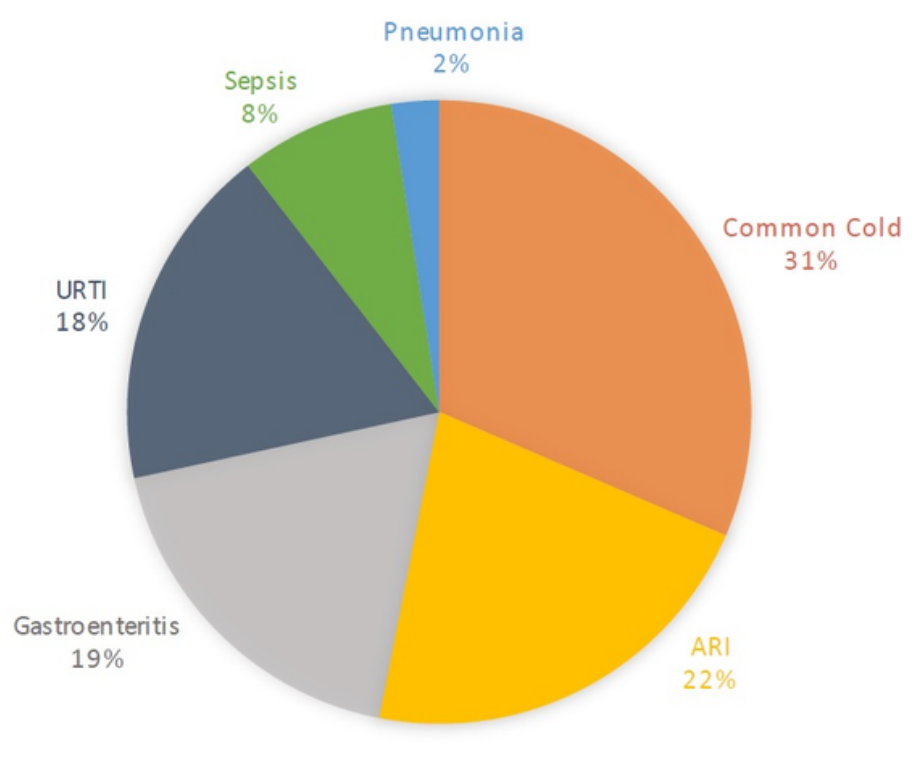

\section{OXYGEN SATURATION <95\%}

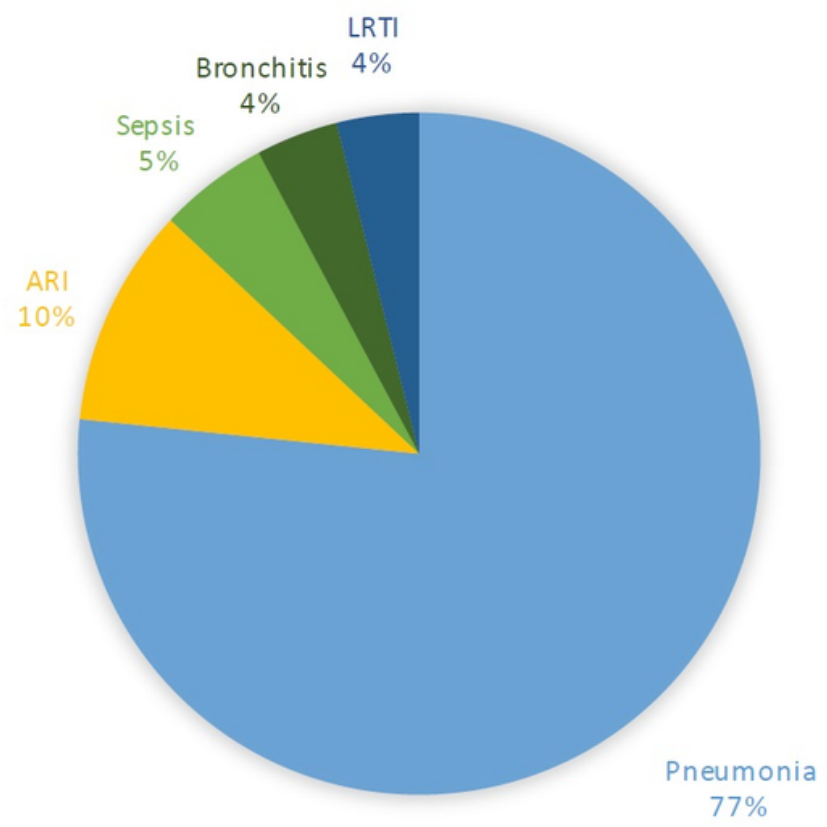

\section{Figure 4}

(Left) Most common diagnoses for patients that received pulse oximeter evaluation with a resulting oxygen saturation $₫ 95 \%$. (Right) Most common diagnoses for patients that received pulse oximeter evaluation with a resulting oxygen saturation $<95 \%$. ARI = Acute Respiratory Infection; URTI = Upper Respiratory Infection; LRTI = Lower Respiratory Infection.

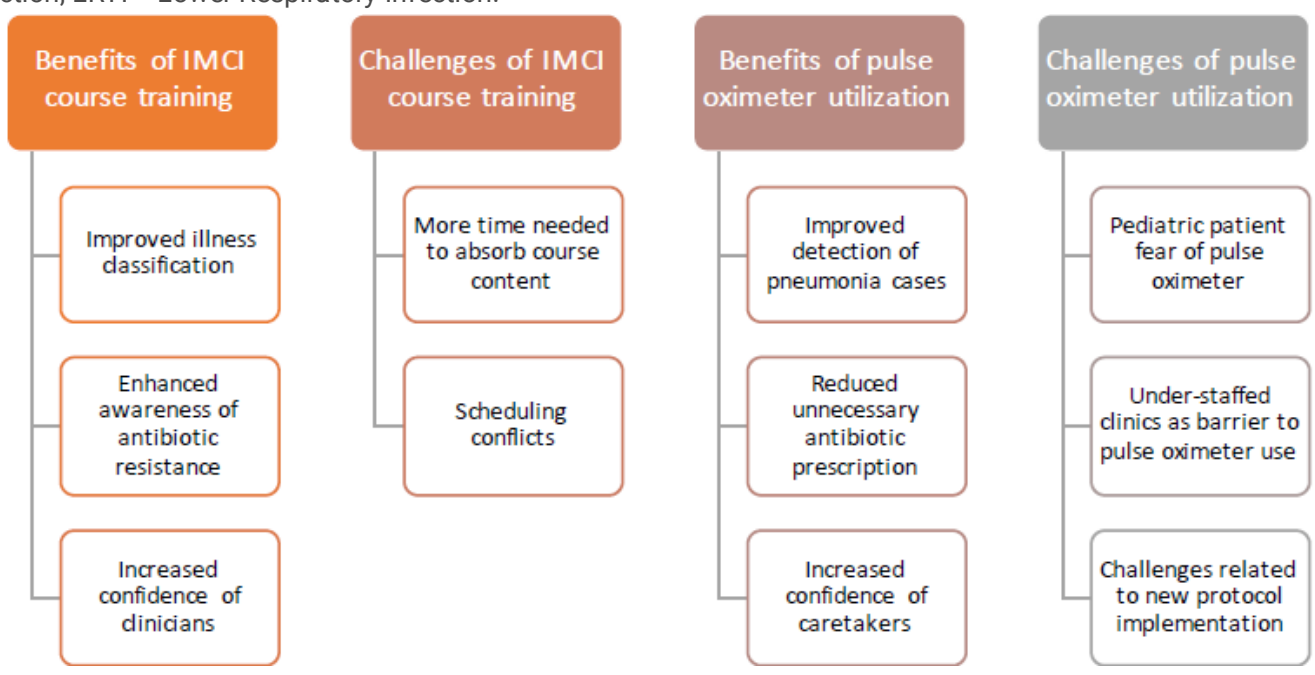

\section{Figure 5}

Key themes from qualitative interviews held with GAIA providers regarding their opinions of the IMCl continued education course training and the pulse oximeter.

\section{Supplementary Files}

This is a list of supplementary files associated with this preprint. Click to download.

- Tables.pdf 